\title{
Assessing the effectiveness of structured teaching on knowledge of hand hygiene among healthcare workers
}

\author{
Sanjiv Ahuja*, Anita Pandey \\ Subharti Medical College, Meerut, Uttar Pradesh, India
}

\section{Introduction}

Health-care associated infections (HCAI) are an important challenge every hospital is dealing with. Hand hygiene is an important element in prevention of HCAIs. The importance of hand washing dates back to 1840s when Ignaz Semmelweis demonstrated that medical students and staff who washed their hands before attending labour room reduced mortality rate from $13-18 \%-2 \% .{ }^{1}$ In mid-1800's Florence Nightingale also recognised its importance in prevention of infection. ${ }^{2}$ Compliance to hand hygiene is low with a median compliance rate of $40 \%$. $^{3}$ The compliance can be improved by several strategies. One of such strategy was given by World Health Organization (WHO) was "My five moments of Hand Hygiene". These are washing of hands before touching a patient, before clean/aseptic procedure, after body fluid exposure, after touching a patient and after touching patient's surrounding. ${ }^{4}$

Other strategies we have adopted in our hospital for increasing awareness and compliance are infection control classes for different healthcare workers every fortnight from October 2014, exhibition on strengthening infection control network on 9th December 2014. To assess effectiveness of structured teaching we initiated assessment of hand hygiene among HCWs by a pre-test and post-test questionnaire which was distributed to them on World hand hygiene day.

\section{Materials and methods}

This study was a descriptive cross-sectional survey carried out on 5th May 2017 (World Hand Hygiene Day) to assess the knowledge of hand hygiene among residents, nurses, nursing students and caregivers (hospital attendants, patient's relatives) in a tertiary care hospital in Western Uttar Pradesh. The MBBS students were divided in groups of 20 students each. These groups of students were first trained by faculty of Microbiology and were posted in respective sections of the hospital where they were accompanied and constantly monitored by the faculty throughout the training. The students were trained vigorously first, with the help of a lecture and demonstration on Hand hygiene followed by hands-on training to each student before being sent for on-site training. The students divided into different groups were posted in different wards mainly in medicine ward, paediatrics, obstetrics \& gynaecology ward, ophthalmology, ear, nose \& throat (ENT) ward, private ward, psychiatry ward and cath lab. As far as ICUs \& Emergency is concerned the compliance for Hand hygiene in our Hospital is better due to constant monitoring by our Infection control team. Our aim here was to target the training of HCW's in the wards as this area is often neglected. Verbal consent was taken from those who volunteered to participate.

A set of 14 questions were given to the participants by the principal investigator as pre-test structured questionnaire. Following which, the HCWs were given training regarding various aspects of hand hygiene for example; about the need of hand hygiene, proper indications of hand washing, hand scrub, five moments of hand rub as recommended by WHO, ideal time for hand wash, hand rub \& hand scrub, proper procedure for hand wash, hand rub and hand scrub and whether gloves are a substitute for hand hygiene, side effects of repeated hand washing and how it can be prevented. After training, the same set of questions was given to the same group of participants as post-test questionnaire. The score was counted. Participation was voluntary and whoever scores the highest in post-test questionnaire received big round of applause and some groups even gave cash price to the best scorer in post-test.

The questions could be answered as yes, no or not sure. For scoring, 1 marks was given for each correct response and 0 marks were given for each incorrect or not sure response indicating poor level of knowledge. A score of $90 \%$ and above was considered as excellent, $75-90 \%$ as good, $50-74 \%$ as moderate and below $50 \%$ was taken as poor.

\subsection{Statistical analysis}

SPSS software version 19.0 (IBM, SPSS statistics) was used for statistical analysis. Chi-square test was performed for data analysis. The pvalues below 0.05 were considered to be significant.

\subsection{Ethics}

Ethical approval was obtained from the Ethics committee of the hospital.

Informed verbal consent was taken from the participants prior to administration of questionnaire after explaining them about the

\footnotetext{
* Corresponding author.

E-mail address: ahujasanjiv2011@gmail.com (S. Ahuja).
} 
Table 1

Hand hygiene Questionnaire.

\begin{tabular}{|c|c|c|c|c|}
\hline & & Pre-test responded correctly $(\mathrm{n}=149)$ & Post-test responded correctly $(n=149)$ & $\mathrm{P}$ value \\
\hline 1 & Is hand washing with soap and water more effective than hand rub & $40(26.8 \%)$ & $102(68.4 \%)$ & $<0.001$ \\
\hline 2 & Is hand hygiene recommended before touching a patient & $133(89.3 \%)$ & $` 146(98.0 \%)$ & 0.002 \\
\hline 3 & Is hand hygiene recommended after abdominal examination & $132(88.6 \%)$ & $147(98.7 \%)$ & 0.001 \\
\hline 4 & $\begin{array}{l}\text { Does washing with soap and water causes more contact dermatitis than hand } \\
\text { rub }\end{array}$ & $80(53.7 \%)$ & $127(85.2 \%)$ & $<0.001$ \\
\hline 5 & Is hand washing recommended before inserting cannula & $125(83.9 \%)$ & $142(95.3 \%)$ & 0.001 \\
\hline 6 & Gloves are a substitute of hand washing & $75(50.3 \%)$ & $123(82.5 \%)$ & $<0.001$ \\
\hline 7 & Is hand washing recommended after cleaning the unoccupied bed & $131(87.9 \%)$ & $144(96.6 \%)$ & 0.005 \\
\hline 8 & Is hand washing recommended after emptying a bed pan & $134(89.9 \%)$ & $144(96.6 \%)$ & 0.021 \\
\hline 9 & Hands should be washed with soap and water after hand rub & $82(55.0 \%)$ & $116(77.8 \%)$ & $<0.001$ \\
\hline 10 & Hand hygiene should be done for a minimum of $30 \mathrm{~s}$ & $105(70.5 \%)$ & $142(95.3 \%)$ & $<0.001$ \\
\hline 11 & Rings and wrist watches should be removed before hand hygiene & $136(91.3 \%)$ & $145(97.3 \%)$ & 0.026 \\
\hline 12 & Gloves can be washed and re-used & $125(83.9 \%)$ & $147(98.7 \%)$ & $<0.001$ \\
\hline 13 & Hand rub should be done for soiled hands & $71(47.6 \%)$ & $104(69.8 \%)$ & $<0.001$ \\
\hline 14 & Hands should be dried after hand hygiene & $132(88.6 \%)$ & $145(97.3 \%)$ & 0.003 \\
\hline
\end{tabular}

purpose of the study.

\section{Results}

A total of 14 questions were distributed to 165 HCW's. Out of 165 HCW's, only 149 participated in both pre-test, training \& post-test questionnaire giving a response rate of $90.3 \%$. It was noted that the response rate was much higher in case of HCW's posted in Cath lab (60\% excellent in pre-test test questionnaire) as compared to HCW's in other departments. There was a significant improvement in knowledge after training as seen after comparison of results of post-test questionnaire from its pre-test counter-part [Tables 1 and 2]. A total of 20/ $149(13.4 \%)$ were patient's relative. The post-test knowledge assessment became excellent (55\%) as compared to pre-test (5\%) in patient's relatives. There was a significant difference in the knowledge of residents, nurses and nursing students as compared to caregivers in the pre-test hand hygiene questionnaire. But there was no significant difference in post-test hand hygiene knowledge between residents, nurses, nursing students and caregivers (Nursing attendant \& relatives of patient) except in knowledge regarding which hand hygiene should be used for soiled hands ( Table 3).

\section{Discussion}

Proper hand washing is the single most important, easiest and the least expensive methods for prevention of Healthcare associated infection and antimicrobial resistantce. ${ }^{5-11}$ Nosocomial transmission of infection generally result from breach in infection control guidelines. This can be curtailed mainly by understanding the gaps in knowledge of hand hygiene, training on hand hygiene and further testing of their knowledge and practice of hand hygiene.

In our study the response rate was $90.3 \%$ which was similar to a study done in North Western Nigeria (88.9\%). ${ }^{12}$ In our study all the groups have a moderate knowledge on hand hygiene which was similar to a study conducted by Nair $\mathrm{SS}^{13}$ and Zakeri $\mathrm{H}^{14}$ except that we also

Table 2

Comparison of Pre and Post-test analysis of responses.

\begin{tabular}{llll}
\hline Scores & $\begin{array}{l}\text { Pre-test } \\
(\mathrm{n}=149)\end{array}$ & $\begin{array}{l}\text { Post-test } \\
(\mathrm{n}=149)\end{array}$ & $\begin{array}{l}\text { Chi-square test \& p } \\
\text { value }\end{array}$ \\
\hline $\begin{array}{l}\text { Poor }<7(<50 \%) \\
\text { Moderate } 7-10 \\
(50-75 \%)\end{array}$ & $9(6 \%)$ & $0(0 \%)$ & $105.3, \mathrm{p}<0.001$ \\
$\begin{array}{c}\text { Good } 11-12 \\
(75-90 \%)\end{array}$ & $57(38.2 \%)$ & $45(30.2 \%)$ & \\
$\begin{array}{c}\text { Excellent } 13-14 \\
(>90 \%)\end{array}$ & $13(8.7 \%)$ & $90(60.4 \%)$ & \\
\hline
\end{tabular}

tested knowledge of caregivers. In our study $88.6 \%$ had knowledge of drying their hands after hands hygiene which increased to $97.3 \%$ after training. This was less than a study done by Timothy \& Ifeoma which reported that $82.5 \%$ of respondents dry their hands after handwashing. ${ }^{15}$ In our study $6 \%$ had the score of $<50 \%$ (poor) and $46.9 \%$ had a good knowledge score (i.e the score $>75 \%$ ), which was higher than a study by Mashhad, Iran. ${ }^{14}$

On post-test scoring, none of the participants scored $<50 \%$ and $90.6 \%$ of them had a good or excellent knowledge score. In our study there was a significant positive relationship between education and knowledge of HCW's regarding Hand hygiene which was mitigated by training. Our study was similar to Suchitra et al., ${ }^{16}$ who reported a positive relationship between education and knowledge of all HCW categories but Duggan et al. ${ }^{17}$ reported a negative relationship between education and hand washing compliance. Van De Mortel et al. found that in nursing students hand hygiene was significantly higher than that of medical students. ${ }^{18}$ In our study (113/129) $87.6 \%$ of HCW's had knowledge of hand hygiene before inserting a cannula (i.e. invasive procedure) but that knowledge increased to (124/129)96.1\% after training given to HCW's which is less than the study done from Nepal (98.5\%). ${ }^{19}$ A total of (119/129) 92.2\% HCW's dried Hands after hand hygiene which is similar to the study from Nepal $(90.9 \%)^{19}$ but larger than a study done by Tinothy \& Ifeoma, ${ }^{15}$ but it in our study it increased to $(127 / 129) 97.7 \%$ after training given to HCW's. Our study for the first time showed that imparting training to all HCW's including patient caregivers can decrease the significant difference in knowledge between caregivers and other HCW's.

Our study showed the important of training sessions on awareness of hand hygiene. These training sessions should be conducted more frequently and there should be a curriculum by MCI that every student of medical, nursing and paramedical college will participate in transferring knowledge of hand hygiene to all HCW's and if possible knowledge should be transmitted to community by celebrating hand hygiene week every year starting from world Hand hygiene day.

The present study had some limitations; i) hand hygiene attitude, practice and compliance were not evaluated. ii) a cross sectional study design was used to collect data on knowledge by pre and post-test testing; a longitudinal study by observation could have helped us better evaluate the data. iii) our sample distribution was not uniform for different healthcare workers and caregivers.

\section{Acknowledgement}

We acknowledge the efforts of the statistician, Mr. Arvind Shukla. 
Table 3

Comparison of Pre-test \& Post-test Response to Knowledge based questions among nurses, doctors and caregivers Hand hygiene Questionnaire.

\begin{tabular}{|c|c|c|c|c|c|c|c|}
\hline & & & $\begin{array}{l}\text { Nursing students } \\
\text { (23) }\end{array}$ & Nurses (47) & $\begin{array}{l}\text { Medical students } \\
\text { (15) }\end{array}$ & $\begin{array}{l}\text { Care-givers } \\
(64)\end{array}$ & $\begin{array}{l}\mathrm{P} \text { value of Pre-test } \\
\text { response }\end{array}$ \\
\hline \multirow[t]{2}{*}{1} & \multirow{2}{*}{$\begin{array}{l}\text { Is hand washing with soap and water more effective than } \\
\text { hand rub }\end{array}$} & Pre-test & $5(21.7 \%)$ & $9(19.1 \%)$ & $7(46.7 \%)$ & $19(29.7 \%)$ & 0.172 \\
\hline & & Post-test & $15(65.2 \%)$ & $30(63.8 \%)$ & $12(80 \%)$ & $45(70.3 \%)$ & 0.6582 \\
\hline \multirow[t]{2}{*}{2} & \multirow[t]{2}{*}{ Is hand hygiene recommended before touching a patient } & Pre-test & $20(86.9 \%)$ & $42(89.4 \%)$ & $14(93.3 \%)$ & $57(89.1 \%)$ & 0.947 \\
\hline & & Post-test & $23(100 \%)$ & $46(97.9 \%)$ & $15(100 \%)$ & $62(96.8 \%)$ & 0.756 \\
\hline \multirow[t]{2}{*}{3} & \multirow[t]{2}{*}{ Is hand hygiene recommended after abdominal examination } & Pre-test & $23(100 \%)$ & $42(89.4 \%)$ & $14(93.3 \%)$ & $53(82.8 \%)$ & 0.142 \\
\hline & & Post-test & $23(100 \%)$ & $47(100 \%)$ & $15(100 \%)$ & $62(96.8 \%)$ & 0.441 \\
\hline \multirow[t]{2}{*}{4} & \multirow{2}{*}{$\begin{array}{l}\text { Does washing with soap and water causes more contact } \\
\text { dermatitis than hand rub }\end{array}$} & Pre-test & $10(43.5 \%)$ & $26(55.3 \%)$ & $7(46.7 \%)$ & $37(57.8 \%)$ & 0.148 \\
\hline & & Post-test & $23(100 \%)$ & $41(87.2 \%)$ & $11(73.3 \%)$ & $52(81.2 \%)$ & 0.084 \\
\hline \multirow[t]{2}{*}{5} & \multirow[t]{2}{*}{ Is hand washing recommended before inserting cannula } & Pre-test & $20(86.9 \%)$ & $44(93.6 \%)$ & $15(100 \%)$ & $46(71.9 \%)$ & 0.021 \\
\hline & & Post-test & $23(100 \%)$ & $47(100 \%)$ & $15(100 \%)$ & $57(89 \%)$ & 0.021 \\
\hline \multirow[t]{2}{*}{6} & \multirow[t]{2}{*}{ Gloves are a substitute of hand washing } & Pre-test & $8(34.8 \%)$ & $24(51.1 \%)$ & $14(93.3 \%)$ & $29(45.3 \%)$ & 0.003 \\
\hline & & Post-test & $14(60.9 \%)$ & $38(80.8 \%)$ & $15(100 \%)$ & $56(87.5 \%)$ & 0.039 \\
\hline \multirow[t]{2}{*}{7} & \multirow{2}{*}{$\begin{array}{l}\text { Is hand washing recommended after cleaning the } \\
\text { unoccupied bed }\end{array}$} & Pre-test & $21(91.3 \%)$ & $41(87.2 \%)$ & $13(86.7 \%)$ & $56(87.5 \%)$ & 0.959 \\
\hline & & Post-test & $23(100 \%)$ & $43(91.5 \%)$ & $15(100 \%)$ & $63(98.4 \%)$ & 0.121 \\
\hline \multirow[t]{2}{*}{8} & \multirow{2}{*}{ Is hand washing recommended after emptying a bed pan } & Pre-test & $19(82.6 \%)$ & $43(91.5 \%)$ & $15(100 \%)$ & $57(89.1 \%)$ & 0.359 \\
\hline & & Post-test & $23(100 \%)$ & $46(97.9 \%)$ & $15(100 \%)$ & $63(98.4 \%)$ & 0.045 \\
\hline \multirow[t]{2}{*}{9} & \multirow[t]{2}{*}{ Hands should be washed with soap and water after hand rub } & Pre-test & $11(47.8 \%)$ & $24(51.1 \%)$ & $14(93.3 \%)$ & $33(51.6 \%)$ & 0.019 \\
\hline & & Post-test & $16(69.6 \%)$ & $33(70.2 \%)$ & $15(100 \%)$ & $52(81.2 \%)$ & 0.065 \\
\hline \multirow[t]{2}{*}{10} & \multirow[t]{2}{*}{ Hand hygiene should be done for a minimum of $30 \mathrm{~s}$} & Pre-test & $15(65.2 \%)$ & $39(83.0 \%)$ & $14(93.3 \%)$ & $37(57.8 \%)$ & 0.006 \\
\hline & & Post-test & $23(100 \%)$ & $46(97.9 \%)$ & $14(93.3 \%)$ & $59(92.2 \%)$ & 0.342 \\
\hline \multirow[t]{2}{*}{11} & \multirow{2}{*}{$\begin{array}{l}\text { Rings and wrist watches should be removed before hand } \\
\text { hygiene }\end{array}$} & Pre-test & $20(86.9 \%)$ & $47(100 \%)$ & $13(86.7 \%)$ & $56(87.5 \%)$ & 0.087 \\
\hline & & Post-test & $23(100 \%)$ & $47(100 \%)$ & $15(100 \%)$ & $60(93.7 \%)$ & 0.141 \\
\hline \multirow[t]{2}{*}{12} & \multirow[t]{2}{*}{ Gloves can be washed and re-used } & Pre-test & $20(86.9 \%)$ & $40(85.1 \%)$ & $14(93.3 \%)$ & $51(79.7 \%)$ & 0.565 \\
\hline & & Post-test & $23(100 \%)$ & $45(95.7 \%)$ & $15(100 \%)$ & $64(100 \%)$ & 0.221 \\
\hline \multirow[t]{2}{*}{13} & \multirow[t]{2}{*}{ Hand rub should be done for soiled hands } & Pre-test & $12(52.2 \%)$ & $20(42.5 \%)$ & $9(60 \%)$ & $30(46.9 \%)$ & 0.657 \\
\hline & & Post-test & $12(52.2 \%)$ & $28(59.6 \%)$ & $9(60 \%)$ & $55(85.9 \%)$ & 0.003 \\
\hline \multirow[t]{2}{*}{14} & \multirow[t]{2}{*}{ Hands should be dried after hand hygiene } & Pre-test & $21(91.3 \%)$ & $46(97.9 \%)$ & $14(93.3 \%)$ & $51(79.7 \%)$ & 0.023 \\
\hline & & Post-test & $22(95.6 \%)$ & $46(97.9 \%)$ & $15(100 \%)$ & $62(96.8 \%)$ & 0.858 \\
\hline
\end{tabular}

\section{References}

1. Best M, Neuhauser D. Heroes and martyns of quality and safety. Ignaz semmelweis and the birth of infection control. Qual Saf Health Care. 2004;13:233-234.

2. Lim F. Why florence Nightingale still matters. Nursing 40;11: 46-47.

3. Erasmus V, Daha T, Brug $\mathrm{H}$ et al. Systematic review of studies on compliance with hand hygiene guidelines in healthcare. Infect Contr Hosp Epidemiol. 31;3:283-294.

4. WHO guidelines on hand hygiene in health care first global patient safety challenge. Clean Care is Safe Care. 2009:1-262http://whqlipdoc.who.int/publications/2009/ 9789241597906 eng.pdf.

5. Guide to implementtation of the WHO multimodal hand hygiene improvement strategy. Available from: http://www.who.int/patientsafety/en/.

6. Mathur P. Hand hygiene: back to the basics of infection control. Indian J Med Res. 2011;134(5):611-620.

7. Boyce JM, Pittet D. Guideline for hand hygiene in health-care settings. Recommendations of the healthcare infection control practices advisory committee and the HICPAC/SHEA/APIC/IDSA hand hygiene task force. Morb Mortal Wkly Rep. 2002;51:1-44.

8. Smith SMS. A review of hand-washing techniques in primary care and community settings. J Clin Nurs. 2009;18:786-790.

9. Infection Control Guidelines. Communicable Disease Report. Canada: Laboratory Centre for Disease Control, Bureau of Infectious Diseases; 1998.

10. Larson E. Skin hygiene and infection prevention: more of the same or different approaches? Clin Infect Dis. 1999;29:1287-1294.

11. Larson E. A causal link between handwashing and risk of infection? Examination of the evidence. Infect Control Hosp Epidemiol. 1988;9:28-36.
12. Iliyasu G, Dayyab FM, Habib ZG, et al. Knowledge and practices of infection control amonghealthcare workers in a Tertiary referral centre in North-Western Nigeria. Ann Afr Med. 2016;15(1):34-40.

13. Nair SS, Hanumantappa R, Hiremath SG, Siraj MA, Raghunath P. Knowledge, attitude and practices of hand hygiene among medical and nursing students at a tertiary health care centre in raichur, India. ISRN Prev Med. 2014;2014:608927https://doi. org $/ 10.1155 / / 2014 / 608927$.

14. Zakeri H, Ahmadi F, Rafeemanesh E, Safeh LA. The knowledge of Hand hygiene among the healthcare workers of two teaching hospitals in Mashhad. Electron Physician. 2017:9(8):5159-5165.

15. Timothy AE, Ifeoma PO. HH knowledge and practices among healthcar providers in tertiary care hospitals, south west Nigeria. Int J Integrated Care. 2013;9:1-10.

16. Suchitra JB, Lakshmi Devi N. Impact of education on knowledge, attitudes and practices among various categories of health care workers on nosocomial infections. Indian J Med Microbiol. 2007;25(3):181-187. https://doi.org/10.4103/0255-0857. 34757.

17. Duggan JM, Hensley S, Khuder S, Papadimos TJ, Jacobs L. Inverse correlation between level of professional education and rate of handwashing compliance in a teaching hospital. Infect Control Hosp Epidemiol. 2008;29(6):534-538. https://doi. org/10.1086/588164.

18. van de Mortel TF, Apostolopoulu E, Petrikkos G. A comparison of the hand hygiene, knowledge, beliefs and practices of Greek nursing and medical students. Am J Infect Contr. 2010;38(1):75-77.

19. Khanal G, Thapa S. Awareness of hand hygiene among healthcare workers in chitwan, Nepal. SAGE. 2017 Oct-Dec:1-7. https://doi.org/10.11772/ 2158244017735141http://journals.sagepub.com/home/sgo. 